\title{
A QUICK GLANCE OF SPLINE WAVELETS AND ITS APPLICATIONS
}

\author{
B Kunwar ${ }^{1}$, V.K Singh ${ }^{2}$, Kanchan Lata Gupta ${ }^{3}$ \\ ${ }^{1}$ Department of Applied Sciences, Institute of Engineering and Technology, Lucknow \\ ${ }^{2}$ Department of Applied Sciences, Institute of Engineering and Technology, Lucknow \\ ${ }^{3}$ Department of Applied Sciences, Institute of Engineering and Technology, Lucknow
}

\begin{abstract}
Polynomial spline wavelets have played a momentous role in the enlargement of wavelet theory. Due to their attractive properties compact support, good smoothness property, interpolation property, they are now provide powerful tools for many scientific and practical problems. As splines have specific formulae in both time and frequency domain, it greatly facilitates their manipulation. This paper is a summary of spline wavelet which started with splines and ends with the applications of spline wavelets. The paper is divided into four sections. The first section contains a brief introduction of splines and the second section is devoted to the discussion of spline wavelet construction via multiresolution analysis (MRA) with emphasis on B-spline wavelet. The underlying scaling functions are B-splines, which are shortest and most regular scaling function. In the third section, some remarkable properties of spline wavelets are discussed. The orthogonality and finite support properties make the spline wavelets useful for numerical applications and also have the best approximation properties among all the known wavelets. And the last section enclose a brief discussion of application of spline wavelets.
\end{abstract}

Keywords: Splines, Multiresolution Analysis, Semi-Orthogonal Bases, Compact Support. $* * *$

\section{INTRODUCTION}

Polynomial splines have a number of attractive properties that make them useful in variety of applications. These features include good smoothness properties, a simple analytical form and the fact that they have convenient representations in terms of simple basis functions. There is a great curiosity in the investigation of compactly supported wavelets. These interests are due to the computational capabilities of spline wavelets and the wide range of their applications. Thanks to some of their exceptional properties and mathematical simplicity, they are also applied and give very good result in various areas of applied sciences in comparison to other known wavelets.

The $\mathrm{m}^{\text {th }}$ order Cardinal B-spline with integer knot sequence $\mathbb{Z}$, defined recursively by

$B_{m}(x)=\left(B_{m-1} * B_{1}\right)(x)=\int_{0}^{1} B_{m-1}(x-t) d t$

where $B_{1}=\chi_{[0,1]}$ is the characteristic function of the unit interval. The Cardinal B-spline has some very interesting properties which make them a perfect fit to use as scaling function in constructing wavelets. These characteristic properties are given in many books on splines. Here the properties are listed in form of a theorem as in [1].

Theorem 1: The $\mathrm{m}^{\text {th }}$ order cardinal B-spline $B_{m}$ satisfies the following properties;

i) Supp $B_{m}=[0, m]$

ii) $B_{m}(x)>0$ for $0<x<m$

iii) $\sum_{k=-\infty}^{\infty} B_{m}(x-k)=1 \quad \forall x$

iv) The Cardinal B-spline $B_{m}$ and $B_{m-1}$ are related by the identity $B_{m}(x)=\frac{x}{m-1} B_{m-1}(x)+\frac{m-x}{m-1} B_{m-1}(x-1)$ v) $B_{m}$ is symmetric with respect to the centre of its support, namely $B_{m}\left(\frac{m}{2}+x\right)=B_{m}\left(\frac{m}{2}-x\right)$

Theorem 2: For any pair of integers $m$ and $j$ with $m \geq 2$, the family $S_{j}=\left\{2^{j} / 2 B_{m}\left(2^{j} x-k\right): k \in \mathbb{Z}\right\}$ is a Riesz basis of subspace $V_{j}^{m} \in L^{2}(R)$ with Riesz bounds $A=$ $\frac{1}{(2 m-1)} \prod_{k=1}^{m-1} \frac{\left(1+\lambda_{k}\right)^{2}}{\left|\lambda_{k}\right|}>0 \quad$ and $\quad B=1$. And also $\sum_{k=-\infty}^{\infty}\left|\widehat{B_{m}}(\omega+2 \pi k)\right|^{2} \geq \sum_{k=-\infty}^{\infty}\left|\widehat{B_{m}}(\pi+2 \pi k)\right|^{2}$

The proof of the above theorem are given in [1].

\section{MULTIRESOLUTION ANALYSIS}

Multiresolution analysis(MRA) is the basic tool for the construction of wavelet bases.

Definition:

A MRA generated by a function $\varphi$ consist of sequence of closed subspaces $V_{j}, j \in \mathbb{Z}$ of $L^{2}(R)$ satisfying

i) $\quad V_{j} \subset V_{j+1}$ for all $j \in \mathbb{Z}$

ii) $\overline{U_{j \in z} V_{j}}=L^{2}(R)$

iii) $\bigcap_{j \in Z} V_{j}=\{0\}$

iv) $f(x) \in V_{j}$ if and only if $f(2 x) \in V_{j+1}$ for all $j \in \mathbb{Z}$

v) There exist a function $\varphi \in V_{0}$ such that $\{\varphi(x-$ $k: k \in \mathbb{Z}\}$ is an orthonormal basis.

The function $\varphi$ defined in the last condition is called scaling function of MRA. For each subspace $V_{j+1}$ there exist an orthonormal compliment $W_{j}$ of $V_{j+1}$ in $V_{j}$ such that,

$W_{j}$ is subspace of $V_{j+1}$

$$
W_{j} \perp V_{j}
$$




$$
V_{j+1}=V_{j} \oplus W_{j}
$$

And

$$
W_{k} \perp W_{l} \text { for all } k \neq l
$$

Hence under condition (i), (ii) (iii), it follows that $L_{2}=$ $\bigoplus_{k \in Z} W_{k}$. The spaces $W_{k}, k \in Z$ are called wavelet spaces of $L_{2}$ relative to the scaling function $\varphi$. A scaling function $\varphi$ must be a function in $L_{2}(R)$ with $\int \varphi \neq 0$. Also since, $\varphi \in V_{0}$ is also in $V_{1}$ and $\left\{\varphi_{1, k}=2^{j} / 2(2 x-k): k \in Z\right\}$ is a Riesz basis of $V_{1}$.

From section 1, it is clear that Cardinal B-spline of order $\mathrm{m}$ generates a MRA of $L_{2}(R)$ in the sense that $V_{k}^{m}=$ $\operatorname{clos}_{L^{2}}\left\{B_{m}\left(2^{k} .-j\right): j \in Z\right\}$. The two scale relation for cardinal B-spline of order $\mathrm{m}$ is written as

$B_{m}(x)=\sum_{k=0}^{m} 2^{-m+1}\left(\begin{array}{c}m \\ k\end{array}\right) B_{m}(2 x-k)$

And Fourier transform of it is,

$\widehat{B_{m}}(\omega)=P(z) \widehat{B_{m}}(\omega / 2)$

where $P(z)=\frac{1}{2} \sum_{k=0}^{m} 2^{-m+1}\left(\begin{array}{c}m \\ k\end{array}\right) z^{k}=\left(\frac{1+z}{2}\right)^{m} ; \quad z=$ $e^{-i \omega / 2}$

$P(z)$ is called the mask of the scaling function.

Theorem 3: A scaling function $\varphi$ with refinement relation

$\varphi(x)=\sum_{-\infty}^{\infty} p_{k} \varphi(2 x-k)$,forms an orthonormal basis[2] only if

$$
|P(z)|^{2}+|P(-z)|^{2}=1
$$

In case of B-spline,

$$
\begin{gathered}
|P(z)|^{2}+|P(-z)|^{2}=\cos ^{2 m} \frac{\omega}{4}+\sin ^{2 m} \frac{\omega}{4} \leq \cos ^{2} \frac{\omega}{4}+ \\
\sin ^{2} \frac{\omega}{4}=1 .
\end{gathered}
$$

Hence, B-spline form orthonormal basis for $m=1$ only. While in general, $B_{m}$ generates semi-orthogonal wavelets with compact supports provided the two scale sequence of the scaling function $B_{m}$ is given by

$$
G(z)=\frac{E_{2 m-1}(z)}{E_{2 m-1}\left(z^{2}\right)} \overline{P(z)},|z|=1
$$

Where $E_{2 m-1}$ is the Euler-Forbenius polynomial of order $2 m-1$ relative to $B_{m}$. Hence the two scale symbol of semiorthogonal wavelet $\psi$ with respect to $B_{m}$ is given by

$$
Q(z)=z^{-1} G(-z) K\left(z^{2}\right)=z^{-1} \frac{E_{2 m-1}(z)}{E_{2 m-1}\left(z^{2}\right)} \overline{P(-z)} K\left(z^{2}\right)
$$

Where $K\left(z^{2}\right)$ is a Laurent series whose coefficient sequence is in $l^{1}$.
Thus the compactly supported semi-orthogonal wavelet $\psi_{m}$ with minimum support that corresponding to the scaling function cardinal B-spline is given in term of its Fourier transform

$\widehat{\psi_{m}}(\omega)=Q(z) \widehat{B_{m}}\left(\frac{\omega}{2}\right)$

where

1) $(-z)^{k}$

$$
Q(z)=\frac{1}{2} \sum_{n} q_{n} z^{n}=\left(\frac{1-z}{2}\right)^{m} \sum_{k=0}^{2 m-2} B_{m}(k+
$$

Hence the cardinal B-spline wavelet is

$\psi_{m}(x)=\sum_{n} q_{n} B_{m}(2 x-n)$

Where $\quad q_{n}=\frac{(-1)^{n}}{2^{m-1}} \sum_{l=0}^{m}\left(\begin{array}{c}m \\ l\end{array}\right) B_{2 m}(n+1-l), \quad n=$ $0, \ldots . .3 m-2$

And has compact support supp $\psi_{m}=[0,2 m-1]$.

\section{PROPERTIES}

In [3], Unser briefly discussed the ten basic properties i,e time-frequency localization, best approximation property, two scale relation etc, holds by spline wavelet family. Here we discussed some more properties holds by B-spline wavelets.

\subsection{Simple Manipulation}

The cardinal B-spline defined as $(n+1)$ fold convolution of characteristic function, thus have simple explicit form, which greatly simplifies its manipulation.

\subsection{Generalized Linear Phase Filtering}

Definition. 1) Let $f \in L^{2}(R)$, then $f$ is said to have generalized linear phase if its Fourier transform satisfies

$$
\hat{f}(\omega)=F(\omega) e^{-i(a \omega+b)} \quad, \quad \text { a.e }
$$

Where $F(\omega)$ is a real-valued function and a, b are real constant.

2) A sequence $\left\{a_{n}\right\} \in l^{1}$ is said to have generalized linear phase if

$$
A\left(e^{-i \omega}\right)=F(\omega) e^{-i(n \omega+b)}
$$

For some real valued function $F(\omega), n \in \frac{1}{2} Z$ and $b \in R$.

From these two definition it is very clear that $B_{m}, m \geq 2$ has generalized linear phase.

\subsection{Spline Interpolation}

The wavelet interpolation function $\bar{\psi}(x)$, is the wavelet function whose scaling function is the interpolation spline-

$$
\bar{\psi}(x)=\sum d_{i} \bar{\varphi}(2 x-i)
$$


The Fourier transform of spline interpolation wavelet is

$$
\hat{\bar{\psi}}(\omega)=\frac{c(-z)}{c\left(z^{2}\right)} Q(z) \hat{\bar{\varphi}}\left(\frac{\omega}{2}\right)
$$

where $\sum d_{i} z^{i}=\frac{c(-z)}{c\left(z^{2}\right)} Q(z)$

\subsection{Oscillating Properties}

The first order B-wavelet $\psi_{1}$ is simply the Haar function,

$$
\psi_{1}=\chi_{[0,1 / 2)}-\chi_{(1 / 2,1]}
$$

Whose oscillating properties will be evident. Let,

$$
f(x)=\sum_{j=0}^{N} c_{j} \psi_{m}(x-j)
$$

Such that, $c_{0} c_{N} \neq 0$, then the number of strong sign changes of $f$ i,e $S^{-}(f)$ lies between $\mathrm{N}+3 \mathrm{~m}-2$ and $2 \mathrm{~N}+3 \mathrm{~m}-2$. Setting $\mathrm{N}=0, S^{-}(f)=3 m-2$.

Thus $\psi_{m}$ has $3 \mathrm{~m}-2$ zeros in the interior of its support $[0,2 \mathrm{~m}]$. And hence, in contrast to the property of "total positivity" of cardinal B-spline $B_{m}$, the cardinal B-wavelet $\psi_{m}$ possesses the property of "complete oscillation".

\section{APPLICATIONS}

Due to the orthonormal bases, wavelets provide fast algorithms in numerical aspects in approximating because of their vanishing moment and small supports, which leads to sparse matrix. Semi-orthogonal compactly supported Bspline wavelets behave better and easier than other wavelets in a bounded interval. For these reason, they are good candidate for solving integral equations.

Splines are smooth and well behaved functions. Splines of degree $n$ are $(n-1)$ times continuously differentiable. As a result, splines have excellent approximation properties. Bsplines and their wavelet counterparts have excellent localization properties so they are good templates for timefrequency signal analysis. The compactly supported Bspline wavelets have been found to be powerful tool in many scientific and practical application including mathematical approximation, the finite element method, image processing and compression and computer-aided geometric design.

\section{CONCLUSION}

Wavelets constructed via multiresolution analysis taking Bspline as scaling function generate orthonormal basis or semi-orthonormal basis depending on order of spline for the wavelet space. Constructed B-spline wavelets have a compact support and explicit formulae which reduces the calculation effort. These wavelets are easier to handle in bounded interval and due to polynomial function have excellent approximation property.

\section{REFERENCES}

[1] C.K. Chui ; An introduction to wavelets, Academic Press, New York, 1992.

[2] I. Daubechies ; Ten lectures on wavelets, CBMS-NSF, Regional conference series in applied mathematics,61, SIAM, Philadelphia,PA, 1992

[3] M. Unser ; Ten good reasons for using spline wavelets, Wavelets applications in signal and image processing V.1997, pp.422-431.

[4] M. Unser, A. Aldraubi ; Spline multiresolutions and wavelet transform

[5] T.-X. He , T. Nguyen ; A note on the Daubechies approach in the construction of spline type orthogonal scaling functions, 2015.

[6] T.-X. He, T. Nguyen ; Construction of spline type orthogonal scaling function and wavelets, Journal of applied Functional analysis, Eudoxus Press, LLC, vol10, 2015.

[7] W. Freeden , M. Gutting ; Constructive Approximations: splines and wavelets, 2013.

[8] A. A. Hemmat, Z. Rahbani; Pantic B-spline wavelets and their application for solving linear integral equations, Iranian journal of science and technology, A1:47-50, 2012.

[9] C. K. Chui , J. Z. Wang ; A cardinal spline approach to wavelets, American mathematical society, vol.113, 1991.

[10] C. K. Chui , J. Z. Wang ; An analysis of cardinal spline wavelets, Journal of Approximation theory 72, 54-68, 1993. 